\title{
Comparación de distintos métodos para evaluar la función androgénica en el adulto mayor
}

\author{
Claudia Campusano M, Francisca Brusco G, \\ Carmen Campino J, Luis Rodríguez P, Eugenio Arteaga U. \\ Assessment of androgenic decline \\ in the elderly
}

Background: The gold standard to assess androgenic status is the measurement of free testosterone by equilibrium dialysis. However, the estimation of free testosterone using formulas based on the law of mass action can be an adequate standard. Aim: To assess androgenic decline in the elderly by different methods. Material and methods: Free testosterone by radioimmunoassay, total testosterone and steroid binding globulin (SHBG) by automated chemiluminiscence system and estradiol by automated electrochemiluminiscence system were measured in 30 male aged $64 \pm 5$ years (range 60-70), and 25 males aged between 20 and 30 years, as control group; devoid of diseases or drugs that could cause hypogonadism. Free androgen index, free testosterone, biavailable testosterone, and free estradiol were calculated using a formula based on the law of mass action. Results: Fifty seven percent of elderly subjects had hypogonadism, according to calculated free testosterone values. Their total testosterone was on average, $152 \mathrm{nd} / \mathrm{dl}$ lower than in young adults, figure that represents a 3.8 $\mathrm{ng} / \mathrm{dl}$ decline per year. According to total testosterone values, $27 \%$ of elderly males had gonadal incompetence. The correlations between calculated free testosterone and total testosterone was 0,95 and between calculated free testosterone and measured free testosterone was 0,67 . The methods employed overestimated ( $76.7 \%$ of hypogonadism when using the free androgen index) or underestimated (27 and 3\% of hypogonadism, considering total or free testosterone, respectively) the ondrogen decline of the elderly. Among the elderly, 16 or $30 \%$ of subjects had an absolute hypoestrogenism, based on estradiol or calculated free estradiol values, respectively. On average there was a 20 and 30\% reduction of estradiol and calculated free estradiol values in the elderly. Conclusions: Calculated free or bioavailable testosterone values should be used to assess androgen decline in elderly men (Rev Méd Chile 2006; 134: 1123-8).

(Key w ords: Aged; Androgens; Hypogonadism)

Recibido el 20 de octubre, 2005. Aceptado el 14 de marzo, 2006.

Departamento de Endocrinología y Servicio de Laboratorios Clínicos, Escuela de Medicina, Pontificia Universidad Católica de Chile, Santiago, Chile.

Correspondencia a: Claudia Campusano. Departamento de Endocrinología, Pontificia Universidad Católica de Chile. Lira 85, 5o piso. Santiago, Chile. Fono: 56-2-3543095. Fax: 56-2-6385675. E mail: ccampusa@med.puc.cl 
$\mathrm{E}^{1}$ envejecimiento masculino se acompaña de una declinación progresiva de la testosterona plasmática, fenómeno denominado con la sigla ADAM (Androgen Decline in the Aging Male). Esta declinación es lineal, de 1 a $2 \%$ por año y comienza

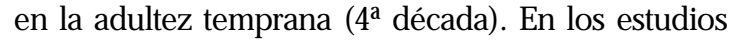
prospectivos, el descenso de la testosterona total ha oscilado entre 3,4 y $11 \mathrm{ng} / \mathrm{dl}$ por año1,2. Este fenómeno provoca que, a medida que envejecen, un porcentaje mayor de hombres sin otra patología concomitante, alcance valores de andrógenos en el rango de hipogonadismo. El porcentaje de hipogonadismo por ADAM es variable de un estudio a otro debido, entre otras razones, a las diferentes poblaciones estudiadas, a la fijación arbitraria de rangos de normalidad y a la diferencia en los métodos utilizados para evaluar el status androgénico ${ }^{1,2}$. Algunos estudios utilizan la testosterona total ${ }^{1,2}$, otros la testosterona libre medida por RIA ${ }^{3}$ o el índice de andrógenos libres (IAL) ${ }^{1}$; sin embargo, todas estas determinaciones tienen una mala correlación con la testosterona libre medida por equilibrio de diálisis, considerada como patrón de oro de la evaluación androgénica. Esta última es una técnica compleja y de difícil aplicación en la práctica clínica. Veurmelen y cols, demostraron, en 1999, que el cálculo de la testosterona libre, basándose en la ley de acción de masas, tiene una correlación casi exacta con el patrón de oro ${ }^{4}$. Esta fórmula incluye a la testosterona total y a diferentes proteínas transportadoras, pero su uso no se ha generalizado y hasta ahora no se ha aplicado en los estudios de ADAM.

\section{SujetOS Y MÉTODO}

Sujetos. Se estudiaron 30 varones entre 60 y 70 años de edad, sin patologías médicas de importancia ni medicamentos que interfirieran con la función gonadal. Se les practicó anamnesis y examen físico a todos ellos y se excluyeron los que tuvieran algún hallazgo que pudiera interferir con las variables a evaluar. Todos tenían TSH y prolactina dentro de rangos normales. Controles: Como grupo control se estudió a 25 varones sanos entre 20 y 30 años, en quienes se determinaron los valores de normalidad de las distintas variables androgénicas y estrogénicas. A todos ellos se les realizó examen clínico, hemograma, perfil bioquímico, TSH y prolactina, a fin de excluir patologías que interfieren en el eje gonadal.

El cálculo del tamaño de la muestra para el grupo de adultos mayores se eligió de acuerdo a los datos obtenidos en los sujetos controles jóvenes que tienen un promedio $\pm \mathrm{DE}$ de testosterona total de 589,4 $\pm 149 \mathrm{ng} / \mathrm{dl}$; así, para encontrar una disminución mínima significativa de $25 \%$ en los adultos mayores se requería de un número igual a 30 en el grupo de estudio.

Métodos. Los sujetos concurrieron a la sala metabólica de la Escuela de Medicina de la Pontificia Universidad Católica de Chile entre 8:00 y 9:00 h, en ayunas, para toma de muestra de sangre para la determinación de testosterona total (TT, Quimioluminiscencia, ACS Centauro, Bayer), testosterona libre (TL, RIA, Coat a Count, DPC), hormona luteinizante ( $\mathrm{LH}$, Quimioluminiscencia, ACS Centauro, Bayer), estradiol (Electroquimioluminiscencia, Elecsys, Roche), sex hormone binding globulin (SHBG, Quimioluminiscencia en fase sólida, Immulite 2000, DPC).

Análisis. El valor promedio - 2DS del grupo control se consideró como el límite inferior de la normalidad para las determinaciones de TT, TL, índice de andrógenos libres (IAL), testosterona libre calculada (TLC), testosterona biodisponible calculada (T Bio C), estrodiol total (ET) y estrodiol libre calculada (ELC).

Se calculó el número de adultos mayores que presentaban valores de cada uno de estos parámetros, bajo la normalidad establecida en el grupo control y se consideró como portador de ADAM aquellos que tenían TT, TL, IAL, TLC y T Bio C, bajo dicho rango.

Por último, la declinación androgénica se acompaña simultáneamente de una caída de estrógenos ${ }^{3}$, la cual está sujeta a las mismas controversias metodológicas del ADAM y cuyo rol biológico es mucho menos claro.

El objetivo de este trabajo fue caracterizar los cambios de los niveles androgénicos y estrogéni$\cos$ en relación al envejecimiento masculino y comparar distintos métodos de laboratorio para evaluar dicha declinación hormonal.

Para la determinación de testosterona libre calculada (TLC) se utilizó la siguiente fórmula de acuerdo a datos previamente publicados ${ }^{4}$ :

$\mathrm{TLC}(\mathrm{moles} / \mathrm{L})=\frac{([\mathrm{T}]-(\mathrm{N} x[\mathrm{TLC}])}{\left(\mathrm{K}_{\mathrm{T}}[\mathrm{SHBG}-\mathrm{T}+\mathrm{N} \mathrm{x} \mathrm{TLC}]\right)}$ 
Donde:

$\mathrm{T}=$ Concentración de testosterona total expresada en moles/L (ng/dl x 34,67 = pmol/L).

$\mathrm{K}_{\mathrm{T}}=1 \times 10^{9} \mathrm{~L} / \mathrm{mol}$, corresponde a la constante de asociación de testosterona a SHBG.

$\mathrm{N}=\mathrm{K}_{\mathrm{a}} \times \mathrm{C}_{\mathrm{a}}+1$ donde $\mathrm{K}_{\mathrm{a}}=3,6 \times 10^{4} \mathrm{~L} / \mathrm{mol}$ representa la constante de afinidad de testosterona a albúmina expresada en $\mathrm{mol} / \mathrm{L}$ y $\mathrm{C}_{\mathrm{a}}$ representa la concentración de albúmina expresada en $\mathrm{mol} / \mathrm{L}$ (peso molecular de la albúmina $=69.000 \mathrm{~g}$ ).

SHBG = Sex Hormone Binding Globulin en moles/L

La ecuación anterior se resuelve como una ecuación matemática de segundo grado cuya solución es:

$$
\mathrm{TLC}=\frac{\sqrt{\left(\left(\left[\mathrm{K}_{\mathrm{T}} \times \mathrm{SHBG}\right]-\left[\mathrm{K}_{\mathrm{T}} \times \mathrm{T}-\mathrm{N}\right]\right)^{2}+4 \mathrm{~N} \times \mathrm{K}_{\mathrm{T}} \times \mathrm{T}\right.}}{2 \mathrm{~N} \times \mathrm{K}_{\mathrm{T}}}
$$

Esta ecuación, como toda ecuación de segundo grado tiene 2 soluciones, dependiendo si el resultado bajo la raíz cuadrada se considera con signo positivo o con signo negativo. En este caso particular, se debe considerar sólo el valor con signo positivo, porque de lo contrario se obtendría una concentración de TLC con signo negativo, que no tiene sustento biológico.

La testosterona biodisponible calculada se obtiene con la fórmula siguiente ${ }^{4}$ :

T Bio C $(\mathrm{mol} / \mathrm{L})=$ TLC $(\mathrm{mol} / \mathrm{L})+$

Testosterona unida a albúmina (mol/L)

Testosterona unida a albúmina $(\mathrm{mol} / \mathrm{L})=\mathrm{Ka} \mathrm{x}$ Ca x TLC (mol/L)
La International Society for the Study of the Aging Male (ISSAM) creo una página web (http:// www.issam.ch/) donde se puede calcular, en forma rápida, la testosterona libre calculada y biodisponible, conociendo los valores de testosterona total, SHBG y albúmina.

El cálculo para el estradiol libre, expresado en moles $/ \mathrm{L}(\mathrm{pg} / \mathrm{ml} \times 3,671=\mathrm{pmol} / \mathrm{L})$ se realiza de la misma manera que la testosterona libre, sólo cambian las constantes de afinidad de estradiol a albúmina y de estradiol a SHBG, cuyos valores se muestran a continuación:

Constante de asociación de estradiol a SHBG = $4 \times 10^{8} \mathrm{~L} / \mathrm{mol}^{5}$.

Constante de asociación de estradiol a albúmina $=3,7 \times 10^{4} \mathrm{~L} / \mathrm{mol}^{5}$.

Todos los sujetos firmaron un consentimiento informado de acuerdo a las normas establecidas por el Comité de Ética de la Facultad de Medicina de la Pontificia Universidad Católica de Chile.

\section{RESULTADOS}

Todos los resultados se expresan como promedio \pm DS. El grupo control tenía $24,6 \pm 3,1$ años de edad y un IMC de $24,8 \pm 2,1 \mathrm{~kg} / \mathrm{m}^{2}$. El grupo de adultos mayores tuvo una edad promedio de $64,4 \pm 3,5$ años y un IMC de $26,8 \pm 2,76 \mathrm{~kg} / \mathrm{m}^{2}$.

La Tabla 1 muestra la correlación de los distintos métodos para valorar el estado androgénico con la TLC y con la T Bio C, evidenciando una excelente correlación con la testosterona total. Al analizar la testosterona libre medida por RIA y el

\section{Tabla 1. Correlación de los valores de andrógenos con la testosterona libre calculada en los sujetos jóvenes}

\begin{tabular}{|lrlrrrrr|}
\hline & Media \pm DE & & $r$ & $p$ & & $r$ & $p$ \\
\hline TT ng/dl & $589,4 \pm 149$ & TT vs TLC & 0,948 & $<0,0001$ & TT vs TBio C & 0,939 & $<0,001$ \\
TL ng/dl & $2,1 \pm 0,51$ & TL vs TLC & 0,665 & $<0,001$ & TL vs Tbio C & 0,015 & \\
SHBG nM/l & $21,3 \pm 5,59$ & & & & & & \\
TLC ng/dl & $15,1 \pm 3,4$ & & & & & & \\
T Bio ng/dl & $381,4 \pm 91,5$ & T Bio C vs TLC & 0,992 & $<0,0001$ & & \\
IAL & $98,7 \pm 21,9$ & IAL vs TLC & 0,633 & $<0,003$ & IAL vs TBioC & 0,780 & $<0,05$ \\
Albúmina g/dl & $4,60 \pm 0,16$ & & & & & & \\
\hline
\end{tabular}

TT: Testosterona Total; TL: Testosterona Libre; TLC: Testosterona libre calculada; T Bio C: Testosterona Biodisponible calculada; SHBG: Sex Hormone Binding Globulin; IAL: Índice de andrógenos libres. 
índice de andrógenos libres, la correlación, aunque significativa, es marcadamente menor.

La Tabla 2 muestra los resultados de las variables androgénicas y estrogénicas en ambos grupos. Respecto a las primeras, se observa que en los adultos mayores hubo una disminución promedio significativa de $26 \%$ en TT, $29 \%$ en TL, $60 \%$ en IAL, $51 \%$ en la T Bio C y 47\% en TLC.

El porcentaje de hipogonadismo en la población mayor estudiada fue de $26,7 \%$ si se consideraba el valor de TT, $56,6 \%$ con TLC, $50 \%$ con T Bio C, 3,3\% con TL y $76,7 \%$ con IAL. La SHBG fue significativamente mayor en el grupo de estudio que en los controles jóvenes ( $p<0,01$ ). La LH tuvo un valor de $4,4 \pm 2,7 \mathrm{mUI} / \mathrm{ml}$ y en todos los sujetos se encontraba en rangos normales.

Respecto a las variables estrogénicas, se observa que el grupo en estudio tiene un estado de hipoestrogenismo con descenso promedio de $20 \%$ en ET y 30\% en ELC. Existe hipoestrogenismo absoluto en $16,7 \%$ de los pacientes si se considera ET y en $30 \%$ al usar ELC.

\section{DisCUSIÓN}

El déficit androgénico en los adultos mayores ha tomado relevancia en los últimos años, pero es aún controvertido cuáles son los mejores criterios de laboratorio para llegar a este diagnóstico. Distintas publicaciones han comunicado prevalencias variables de hipogonadismo, dependiendo del parámetro de laboratorio usado, los valores de referencia de normalidad utilizados e incluso si se considera una medición hormonal aislada o la asociación con un cuadro clínico compatible.

Utilizando la medición de TLC como patrón de oro de la estimación androgénica, encontramos que $56,6 \%$ del grupo de adultos mayores presentaba déficit androgénico o ADAM. La T Bio $\mathrm{C}$ nos muestra una incidencia similar. Sin embargo, no es posible extrapolar esta frecuencia de hipogonadismo a toda la población chilena, dado que nuestro grupo de estudio no era necesariamente representativo de la población general, por el hecho de ser soluntarios»y no seleccionados en forma aleatoria. Además, debe considerarse que el grupo control fue de un rango etario muy estrecho y ello pudo haber elevado los puntos de corte de normalidad de los andrógenos. A pesar de lo anterior, este resultado representa una aproximación inicial al problema del déficit androgénico en Chile y nos permite sospechar la magnitud de dicho problema en nuestra población.

En nuestro estudio, la TT cayó $152 \mathrm{ng} / \mathrm{dl}$ en 4 décadas, lo que corresponde a 3,8 ng/dl por año (o aproximadamente 7 a $10 \%$ anual), similar a lo reportado por el estudio longitudinal de envejeci-

\section{Tabla 2. Variables androgénicas y estrogénicas en controles y adultos mayores y porcentaje de hipogonadismo e hipoestrogenismo}

\begin{tabular}{|lrrrr|}
\hline & $\begin{array}{r}\text { Controles } \\
(\mathrm{n}=25)\end{array}$ & $\begin{array}{r}\text { Adultos } \\
(\mathrm{n}=30)\end{array}$ & $\begin{array}{r}\text { Valor mínimo } \\
\text { normal** }\end{array}$ & $\begin{array}{r}\mathrm{n} \text { (\%) de adultos } \\
\text { bajo el límite inferior }\end{array}$ \\
\hline TT $(\mathrm{ng} / \mathrm{dl})$ & $589,4 \pm 149$ & $437 \pm 198^{*}$ & 290,4 & $8(26,7 \%)$ \\
TL $(\mathrm{ng} / \mathrm{dl})$ & $2,1 \pm 0,51$ & $1,5 \pm 0,3^{*}$ & 1,1 & $1(3,3 \%)$ \\
IAL & $98,7 \pm 21,9$ & $39,2 \pm 15,6^{*}$ & 54,9 & $23(76,7 \%)$ \\
TLC $(\mathrm{ng} / \mathrm{dl})$ & $15,1 \pm 3,4$ & $8,0 \pm 3,4^{*}$ & 8,3 & $17(56,6 \%)$ \\
T Bio C $(\mathrm{ng} / \mathrm{dl})$ & $381,4 \pm 91,5$ & $188,1 \pm 80^{*}$ & 198,4 & $15(50 \%)$ \\
ET $(\mathrm{pg} / \mathrm{ml})$ & $31,9 \pm 6,4$ & $25,8 \pm 6,9^{*}$ & 19,1 & $9(16,7 \%)$ \\
ELC $(\mathrm{pg} / \mathrm{ml})$ & $0,93 \pm 0,19$ & $0,65 \pm 0,17^{*}$ & 0,55 & \\
SHBG $(\mathrm{nmol} / \mathrm{l})$ & $21,3 \pm 5,6$ & $40,7 \pm 16,2^{*}$ & & \\
Albúmina $(\mathrm{g} / \mathrm{dl})$ & $4,60 \pm 0,16$ & $4,32 \pm 0,17$ & & \\
\hline
\end{tabular}

TT: Testosterona Total; TL: Testosterona Libre; IAL: Índice de andrógenos libres; TLC: Testosterona libre calculada; T Bio C: Testosterona biodisponible calculada; ET: Estradiol total, ELC: Estradiol libre calculado; SHBG: Sex Hormone Binding Globulin; *p $\varangle 0,05$ al comparar promedios de jóvenes y adultos mayores con test de $t$ de Student no pareado. *El límite inferior es el promedio menos 2 SD del grupo control. 
miento de Baltimore que demostró una declinación de $3,2 \mathrm{ng} / \mathrm{dl}$ por año ${ }^{1}$. Con la medición de TT, el $26,7 \%$ del grupo mayor quedó en rango de hipogonadismo, cifra superior al 19\% de hipogonadismo reportado por Harman et al en varones entre 60 y 70 años ${ }^{1}$. Los datos del «Massachusetts Male Aging Study», que estudió algo más de 1.900 sujetos, estimó una prevalencia basal de déficit androgénico de $6 \%$ y en el seguimiento de esta cohorte dicho valor aumenta a 12,3\%. También mostró que los niveles de testosterona total disminuyen $1 \%$ a $2 \%$ por año desde la quinta década ${ }^{6}$.

Nuestra alta prevalencia de ADAM, está dada, al menos en parte, porque en esta evaluación sólo consideramos valores de laboratorio y no la asociación con una sintomatología relacionada, sumado a las características de los grupos analizados por nosotros que ya comentamos. Las obvias diferencias entre diversos estudios plantean la necesidad de fijar los rangos de la normalidad para tener datos comparables, ya que muchos estándares están hechos con población que abarca edades avanzadas, contaminando la normalidad» con el fenómeno a estudiar, ya que al incluir sujetos que posean valores de testosterona que ya han empezado a declinar, el promedio obviamente será inferior.

Los diferentes métodos para determinar el status androgénico de nuestro grupo de estudio tuvieron una correlación significativa con TLC, pero en magnitud diferente; la TT fue la mejor correlacionada $(r=0,98)$ seguido por TL e IAL (Tabla 1). Sin embargo, de los 3 métodos estudiados, uno sobreestima $(76,7 \%$ de ADAM si se considera IAL), y dos subestiman $(26,7 \%$ y $3,3 \%$ de ADAM si se considera TT o TL, respectivamente) la frecuencia de hipogonadismo en el adulto mayor. Lo más notorio es el caso de la TL, método muy utilizado en algunos medios, pero que es claramente inadecuado para valorar el status androgénico de los hombres. Esta medición no corresponde a un método de equilibrio de diálisis. Se basa en la medición por RIA y no ha mostrado buena correlación con testosterona libre por equilibrio de diálisis ni biodisponible 7 .

Es interesante destacar que la medición de TT no permite diagnosticar a la mitad de los casos de hipogonadismo de nuestro estudio. Ello no depende tanto de la técnica de medición de TT como al hecho que lo que se mide es la sumatoria de testosterona libre y la unida a albúmina (ambas biodisponibles) y testosterona unida a SHBG (no biodisponible); esta última representa la fracción mayoritaria de testosterona. Dado que con la edad existe un incremento de SHBG (en nuestro grupo de estudio este parámetro se duplicó), habrá un aumento proporcional de TT, pero a expensas de mayor fracción no biodisponible. Esto enfatiza la necesidad de incorporar la medición de SHBG en la evaluación del status androgénico. Por ello, pareceńa razonable utilizar el IAL, que corrige el valor de testosterona por el valor de SHBG y que ha demostrado gran utilidad en la valoración de los trastomos androgénicos en las mujeres. Sin embargo, en nuestro estudio se observa que el IAL sobreestima en 50\% la frecuencia de hipogogandismo, lo cual hace que este método no sea recomendable en la evaluación del hipogonadismo masculino.

En los últimos años, las técnicas de mediciones hormonales han experimentado un importante avance, pero en el caso de las mediciones de testosterona aún se plantean dificultades por la heterogeneidad de las técnicas de medición. La testosterona total, medida por RIA, es una técnica estable y precisa pero muchos de los inmunoensayos más recientes no usan testosterona como estándar, sino que análogos de ella y existe menos validación clínica de estos métodos. Esto ha llevado a que se cambien los valores normales de acuerdo al fabricante de cada método ya que algunos subestiman y otros sobreestiman las mediciones $^{8}$. El análisis de estos autores sugiere que las dificultades se presentan especialmente en la medición de testosterona en las mujeres o en niños, en los que las concentraciones son menores. Otras publicaciones sugieren que las variaciones de los métodos automatizados de medición de andrógenos (no RIA) pudieran afectar el diagnóstico de los hipogonadismos leves como el de la andropausia, siendo necesario plantear que cada laboratorio establezca sus propios rangos de referencia, ya que aunque se use el mismo kit o método automatizado se pueden detectar variaciones entre laboratorios de hasta $23 \% 8,9$.

Comercialmente, existen ensayos para medir testosterona libre y biodisponible (pero esta técnica es engorrosa ya que requiere precipitación con sulfato de amonio). La medición por equilibrio de diálisis es considerado como el patrón de oro y revela que las mediciones de los kits comerciales tienen una menor precisión. 
Teniendo como base estas consideraciones técnicas, debemos considerar además, la variabilidad individual de los niveles de testosterona en un mismo individuo, especialmente dado el ritmo circadiano que tiene la testosterona sérica. Este hecho complica aún más los criterios para hacer el diagnóstico de hipogonadismo en un paciente individual, especialmente el relacionado con el envejecimiento.

Los intentos por medir la testosterona realmente libre concluyeron en que el método ideal es por equilibrio de diálisis, sin embargo es una técnica engorrosa poco aplicable a la práctica clínica, pudiendo asimilarse a la testosterona libre calculada por la ley de acción de masas ${ }^{4}$. Además, el IAL

\section{REFERENCIAS}

1. Harman M, Metter J, Tobin J, Pearson J, Blackman M. Longitudinal effects of aging on serum total and free testosterone levels in healthy men. J Clin Endocrinol Metab 2001; 86: 724-31.

2. Morley JE, Kaiser FE, Perry HM $3^{\mathrm{RD}}$ et al. Longitudinal changes in testosterone, luteinizing hormone, and follicle-stimulating hormone in healthy older men. Metabolism 1997; 46: 410-3.

3. Denti L, Pasolini G, Sanfelici L et al. Aging-related decline of gonadal function in healthy men: Correlation with body composition and lipoproteins. J Am Geriatr Soc 2000; 48: 51-9.

4. Vermeulen A, Verdonck L, Kaufman J. A critical evaluation of simple methods for the estimation of free testosterone in serum. J Clin Endocrinol Metab 1999; 84: 3666-72.

5. Rosenfield RL, MoL GW JR. The role of the proteins in the distribution of plasma androgens and estradiol. In Molinatti G, Martini L and James VHT (eds) Androgenization in women. Raven Press, New York 1983, pp 25-45.

6. Araujo AB, O’Donnell AB, Brambila DJ, Simpson W, Longcope C, Matsumoto AM et al. Prevalence and incidence of androgen deficiency in middleaged and older men: estimates from the Mas- pierde correlación a medida que los niveles de SHBG aumentan ${ }^{10}$.

En conclusión, en la evaluación del estado androgénico de los hombres mayores, sugerimos utilizar la testosterona libre calculada y testosterona biodisponible calculada, que son métodos matemáticos que requiere contar con tres mediciones ampliamente disponibles en nuestro medio (testosterona total, albúmina y SHBG). En caso de no contar con dicho método sugerimos simplemente medir testosterona total, dado que a pesar de ser un método que subdiagnostica el problema del hipogonadismo, es más certero que la medición del TL por RIA o el cálculo de IAL, los que creemos que deben ser desechados de la práctica clínica.

sachusetts Male Aging Study. J Clin Endocrinol Metab 2004; 89: 5920-6.

7. Christ-Crain M, Meier C, Huber P, Zimmeri L, Trummier M, MÜLER B. Comparison of different methods for the measurement of serum testosterone in the aging male. Swiss Med Wkly 2004; 134: 193-7.

8. Wang C, Catun DH, Demers LM, Starcevic B, SwERDLOFF RS. Measurement of Total Serum Testosterone in Adult Men: Comparison of Current Laboratory Methods Versus Liquid Chromatography-Tandem Mass Spectrometry. J Clin Endocrinol Metab 2004; 89: 534-43.

9. Matsumoto AM, Bremner WJ. Serum Testosterone Assays-Accuracy Matters. J Clin Endocrinol Metab 2004; 89: 520-4.

10. De Ronde W, Van Der Schouw YT, Muler M, Grobbee DE, Gooren LG, Pols HA et al. Associations of Sex-Hormone-Binding Globulin (SHBG) with Non-SHBG-Bound Levels of Testosterone and Estradiol in Independently Living Men. J Clin Endocrinol Metab 2005; 90: 157-62.

\section{Agradecimientos}

Los autores deseamos agradecer a Laboratorios Roche los reactivos y las facilidades para realizar la mediciones de estradiol. 